\title{
Diacronie
}

Studi di Storia Contemporanea

$N^{\circ} 14,2$ | 2013

Processo penale, politica, opinione pubblica (secoli XVIII-XX)

\section{Gianfranco Contini, Dove va la cultura europea?}

\section{Luca Zuccolo}

\section{(2) OpenEdition \\ Journals}

Edizione digitale

URL: http://journals.openedition.org/diacronie/295

DOI: $10.4000 /$ diacronie.295

ISSN: 2038-0925

Editore

Association culturelle Diacronie

Notizia bibliografica digitale

Luca Zuccolo, « Gianfranco Contini, Dove va la cultura europea? », Diacronie [Online], Nํ 14, 2 | 2013,

documento 15, Messo online il 01 août 2013, consultato il 23 septembre 2020. URL : http://

journals.openedition.org/diacronie/295; DOI : https://doi.org/10.4000/diacronie.295 


\section{Diacronie}

N. 14 | 2|2013 Processo penale, politica, opinione pubblica (secoli XVIII-XX)

15/

\section{RECENSIONE:}

\section{Gianfranco CONTINI, Dove va la cultura europea?, Macerata, Quodlibet, 2012, 63 pp.}

a cura di Luca ZUCCOLO *

I primi anni del nuovo millennio, la recente crisi finanziaria e sociale che sta coinvolgendo l'emisfero occidentale e il nuovo assetto che si è data l'Europa negli ultimi due decenni pongono all'ordine del giorno una necessaria riflessione sulla cultura europea contemporanea e sul suo futuro. È oggi necessario, più che in altri frangenti, porsi delle domande su quale direzione debba prendere la cultura europea e se sia ancora possibile parlare di uno "spirito europeo" in grado di influenzare e guidare la cultura continentale e non solo nei prossimi anni.

In questa situazione di crisi economico-politica e cambiamento sociale, da più parti sono stati rimessi in discussione i valori tradizionali dell'Occidente e dell'Europa in particolare. Tutto ciò è avvenuto in una contingenza in cui l'Europa si è trovata di fronte ad un rinnovamento radicale della sua forma istituzionale e a nuove sfide socioculturali che ne hanno da più parti messo in discussione le "radici" fondative, se di "radici" si può ancora parlare. Il saggio di Gianfranco Contini, Dove va la cultura europea?, riedito dalla casa editrice Quodlibet nel 2012, si presenta come un ottimo punto di partenza per analizzare l'odierna cultura europea e, soprattutto, interrogarsi sul futuro che vogliamo dare a queste espressioni culturali, non più limitate dalle ristrette frontiere nazionali, ma oramai aperte alla condivisione transnazionale e globale.

Edito per la prima volta nel 1946, sulla rivista di lettere, scienze ed arti «La Fiera letteraria» , questo resoconto del Rencontre internationale tenutosi nel medesimo anno a Ginevra e redatto da un giovane Contini (Domodossola, 4 gennaio 1912 - 1 febbraio 1990) con un tono pacato e attento, rappresenta lo spaccato più chiaro e vivo della cultura europea e del suo spirito all’indomani della Seconda Guerra Mondiale.

${ }^{1}$ Come sottolinea Luca Baranelli nella nota al testo, il testo presentato, in assenza di originali d'autore, è quello apparso su La Fiera Letteraria, I, 30, 31 Ottobre 1946. 
Uno spirito incarnato dalla stessa rivista italiana, la quale, fondata nel 1925 da Umberto Fracchia, dopo un breve intermezzo dovuto alla guerra, riappare proprio nel 1946 con un comitato di redazione di tutto rispetto formato, assieme a Contini, da Corrado Alvaro, Emilio Cecchi e Giuseppe Ungaretti. Una redazione formata con lo scopo di interpretare e rappresentare al meglio proprio quello spirito europeo e moderno che Contini descrive al Rencontre ginevrino, lamentando lo scarso numero di intellettuali italiani presenti:

\section{Troppi pochi italiani}

[...] Che poi ai promotori non sia venuto in mente di affiancare al savio Flora [unico italiano presente, visto il forfait di Croce, $n d r]$ qualche altro italiano, fra i più segnalati per il gusto delle idées générales in aggiunta ai meriti creativi o per il commercio di molte culture (e gli rimaneva solo l'imbarazzo della scelta, Montale o Bacchelli, Vigolo o Alvaro, Burzio o Alberti, per non dire che dei più adatti al Pen Club, e lasciamo stare i filosofi "giovani", da Calogero a Capitini, da Antoni a Bobbio, da Luporini a Del Noce), ciò prova lo squallore della loro informazione per tutto quanto accade fuori di Francia. [...] attorno a Flora gravitavano pochi giornalisti, taluno dei quali, e in specie uno che aveva stimato spiritoso chiedere la parola per insolentirlo, aveva per di più lasciato scandalizzato ricordo [...]. Silone, che s'era messo in lista, preferì ripartire. C'era dunque una lacuna al posto dell'Italia, e non provo nessuna "boria della nazioni" a riconoscere che si sentiva².

Oggi come allora, questo agile e brevissimo scritto pone delle domande sulle strade che l'Europa ha preso e prenderà nel prossimo futuro, ma soprattutto traccia un quadro teorico che vede la cultura strettamente connessa alla politica e in una posizione oramai inscindibile da questa. L’immagine della situazione e della vivacità culturale dell'Europa post bellica riportata da Contini, quale inviato e redattore de «La Fiera letteraria», personificata dal dibattito tra Jasper e Lukács, è facilmente adattabile ai nostri giorni, soprattutto nell'espressione di un dibattito politico che, oltre a non essere più scindibile da quello culturale - in quanto esso stesso produttore primario di cultura - sembra aver ripreso in qualche modo i toni di scontro e opposizione tra una destra e una sinistra contrapposte su opposti fronti. Una chiara dimostrazione di questo legame è rappresentata dallo stesso Gianfranco Contini ${ }^{3}$, il quale coniugò in diversi modi

${ }^{2}$ CONTINI, Gianfranco, Dove va la cultura europea?, Macerata, Quodlibet, 2012, pp. 18-21.

3 La figura di Gianfranco Contini è senza dubbio una delle più importanti e più rilevanti della letteratura e della critica italiane. Attraverso i suoi numerosi scritti sui maggiori scrittori e intellettuali italiani da Dante e Petrarca fino a Montale e Gadda, con i quali ebbe anche un 
politica e letteratura sia nella sua esperienza partigiana - partecipò alla Repubblica partigiana dell'Ossola nelle fila del Partito d'Azione (9 settembre - 22 ottobre 1944) sia nella sua ricca produzione critico-letteraria in cui si riflette la sua concezione della politica come "atto d'amore"4.

Il significato ultimo e l'interesse maggiore di questo breve saggio, nondimeno, al di là dei dati concreti riportati e riproposti come uno spaccato storico della cultura europea, resta la problematizzazione e l'apertura nei confronti del futuro della cultura europea e sul suo necessario bisogno di rinnovamento. Infatti, nel 1946, la nuova frontiera che si prospettava alla cultura europea era il confronto-scontro con il comunismo e le sue teorie socio-politico-culturali, che fanno affermare a Contini:

Mi rincrescerebbe che il mondo si fermasse a Lukács, ma ben maggior rovina sarebbe che si fermasse prima, magari drappeggiandosi dell'ostentazione di questo arresto e non attraversasse una così decisa esperienza5.

Nel nuovo millennio la nuova frontiera che si affaccia di fronte alla cultura europea è l'apertura all'altro, la convivenza con nuove culture su un piano non più colonialistico ma paritario. Una situazione che da tempo l'Europa affronta, ma rispetto alla quale mai come negli ultimi anni si è dimostrata culturalmente propositiva. Una contingenza che potrebbe modificare la frase sopra citata di Contini in questi termini: mi rincrescerebbe che l'Europa si fermasse di fronte alle nuove culture extra-europee, ma ben maggior rovina sarebbe se si fermasse prima, escludendole dalla propria struttura concettuale e materiale.

La sfida che la cultura europea si trova ad affrontare, infatti, è proprio quella di superare il suo atteggiamento colonialista ed orientalista e di accogliere all'interno del proprio seno espressioni culturali anche contrastanti, ma ricche di nuova linfa sia a livello culturale che socio-politico.

Se, come sottolinea Contini nel suo resoconto ginevrino, la politica e la cultura non possono essere disgiunte perché parte di una medesima società e di una medesima espressione sociale, l'Europa non può rifiutare le proposte e le spinte, provenienti e dall'interno e dall'esterno, portate avanti da gruppi sociali non europei.

intenso rapporto epistolare, Contini ha sviluppato nuove teorie critiche ancora oggi rilevanti come il dibattito su monolinguismo-plurilinguismo e la critica delle varianti.

4 ITALIA, Paola, Gianfranco Contini, in Dizionario Biografico degli Italiani, URL: < http://www.treccani.it/enciclopedia/contini-gianfranco_(Dizionario-Biografico)/ > [consultato il 21 febbraio 2013].

5 CONTINI, Gianfranco, op. cit., p. 27. 
Sebbene alcune correnti politiche europee vogliano ripulire da queste nuove esperienze ed espressioni culturali la società e la cultura europee rivendicando le più volte citate e abusate "radici" europee, la maggioranza della società e degli intellettuali europei si batte per una migliore integrazione di queste nuove correnti in grado di rivitalizzare la cultura europea e di farla progredire su di un nuovo livello.

In conclusione, il piacevole saggio di Contini, che all'apparenza potrebbe sembrare un semplice resoconto dei colloqui tenutisi a Ginevra nel 1946 e quindi un mero esercizio di memoria storiografica, si propone in tutta la sua freschezza stilistica e tematica come un ottimo pamphlet per interrogarsi sul nostro presente e ancor di più sul nostro futuro.

La nota al testo di Luca Baranelli e il saggio conclusivo di Daniele Giglioli ${ }^{6}$, uniti alle note biografiche sui partecipanti all'incontro ginevrino, rendono ancor più utile e piacevole il resoconto di Contini, il quale, già nel 1946, riceverà i complimenti da parte di Eugenio Montale, che in una lettera del novembre 1946 scriverà:

Quanta soddisfazione mi ha dato sentir toccare come tu solo puoi fare i punti che più c’importano, nel tuo reportage di Ginevra. Raramente ti eri scoperto così e avevi parlato anche per altri con tanta autorità7.

${ }^{6}$ GIGLIOLI, Daniele, Pedagogia della forma, in CONTINI, Gianfranco, op . cit, pp. 43-57.

7 ISELLA, Daniele (a cura di), Eusebio e Trabucco. Carteggio di Eugenio Montale e Gianfranco Contini, Milano, Adelphi, 1997, pp. 146-147, citato in CONTINI, Gianfranco, op. cit, p. 8. 


\section{* L'autore}

Luca Zuccolo, dottore (PhD) in Storia Contemporanea del SUM di Napoli ha sviluppato una ricerca sulle dinamiche di formazione dell'identità ottomana e di un discorso di tipo patriottico durante il regno di Abdülhamid II (1880-1885) attraverso lo studio di fonti a stampa redatte in lingua francese. Già dottore magistrale in Storia d'Europa (Bologna 2008), si è occupato dello sviluppo della modernità durante l'ultimo secolo dell'Impero Ottomano, del confronto/scontro tra modernità e tradizione in un contesto cosmopolita e allo sviluppo dei movimenti sociali che hanno preparato l'avvento della società turca contemporanea.

URL: < http://www.studistorici.com/2010/12/o1/luca-zuccolo >

\section{Per citare questo articolo:}

ZUCCOLO, Luca, «Recensione: Gianfranco CONTINI, Dove va la cultura europea?, Macerata, Quodlibet, 2012, 63 pp.», Diacronie. Studi di Storia Contemporanea : Processo penale, politica, opinione pubblica (secoli XVIII-XX), 29/08/2013,

URL:< http://www.studistorici.com/2013/08/29/zuccolo_numero_14/ >

Diacronie Studi di Storia Contemporanea $\beta$ www.diacronie.it

Risorsa digitale indipendente a carattere storiografico. Uscita trimestrale.

redazione.diacronie@hotmail.it

Comitato di redazione: Marco Abram - Jacopo Bassi - Luca Bufarale - Alessandro Cattunar - Elisa Grandi - Deborah Paci - Fausto Pietrancosta - Matteo Tomasoni - Luca Zuccolo 